\title{
Correlated Dirac Eigenvalues and Axial Anomaly in Chiral Symmetric QCD
}

\author{
H.-T. Ding $\odot,{ }^{1}$ S.-T. Li $\odot,{ }^{2,1}$ Swagato Mukherjee $\odot,{ }^{3}$ A. Tomiya $\odot,{ }^{4}$ X.-D. Wang $\odot,{ }^{1}$ and Y. Zhang $\oplus^{1, *}$ \\ ${ }^{1}$ Key Laboratory of Quark and Lepton Physics (MOE) and Institute of Particle Physics, Central China Normal University, \\ Wuhan 430079, China \\ ${ }^{2}$ Institute of Modern Physics, Chinese Academy of Sciences, Lanzhou 730000, China \\ ${ }^{3}$ Physics Department, Brookhaven National Laboratory, Upton, New York 11973, USA \\ ${ }^{4}$ RIKEN-BNL Research Center, Brookhaven National Laboratory, Upton, New York 11973, USA
}

(Received 4 November 2020; accepted 25 January 2021; published 22 February 2021)

\begin{abstract}
We introduce novel relations between the derivatives $\left[\partial^{n} \rho\left(\lambda, m_{l}\right) / \partial m_{l}^{n}\right]$ of the Dirac eigenvalue spectrum $\left[\rho\left(\lambda, m_{l}\right)\right]$ with respect to the light sea quark mass $\left(m_{l}\right)$ and the $(n+1)$-point correlations among the eigenvalues $(\lambda)$ of the massless Dirac operator. Using these relations we present lattice QCD results for $\partial^{n} \rho\left(\lambda, m_{l}\right) / \partial m_{l}^{n}(n=1,2,3)$ for $m_{l}$ corresponding to pion masses $m_{\pi}=160-55 \mathrm{MeV}$ and at a temperature of about 1.6 times the chiral phase transition temperature. Calculations were carried out using $(2+1)$ flavors of highly improved staggered quarks with the physical value of strange quark mass, three lattice spacings $a=0.12,0.08,0.06 \mathrm{fm}$, and lattices having aspect ratios 4-9. We find that $\rho\left(\lambda \rightarrow 0, m_{l}\right)$ develops a peaked structure. This peaked structure arises due to non-Poisson correlations within the infrared part of the Dirac eigenvalue spectrum, becomes sharper as $a \rightarrow 0$, and its amplitude is proportional to $m_{l}^{2}$. We demonstrate that this $\rho\left(\lambda \rightarrow 0, m_{l}\right)$ is responsible for the manifestations of axial anomaly in two-point correlation functions of light scalar and pseudoscalar mesons. After continuum and chiral extrapolations we find that axial anomaly remains manifested in two-point correlation functions of scalar and pseudoscalar mesons in the chiral limit.
\end{abstract}

DOI: 10.1103/PhysRevLett.126.082001

Introduction.-The Lagrangian of the $(2+1)$-flavor quantum chromodynamics (QCD) with the physical value of strange quark mass $\left(m_{s}\right)$ and degenerate up and down light quarks possesses $\mathrm{SU}(2)_{L} \times \mathrm{SU}(2)_{R}$ chiral symmetry and $\mathrm{U}(1)_{A}$ axial symmetry in the chiral limit of light quark mass $m_{l} \rightarrow 0$. The chiral symmetry is spontaneously broken in the vacuum and the $\mathrm{U}(1)_{A}$ symmetry is anomalously broken due to quantum interactions. For the physical value of $m_{l}$, the broken chiral symmetry of the QCD vacuum gets approximately restored through a smooth crossover at a high temperature $T \simeq 156 \mathrm{MeV}$ [1-6], and for $m_{l} \rightarrow 0$ the restoration takes place via a chiral phase transition at a temperature $T_{c}=132_{-6}^{+3} \mathrm{MeV}$ [7].

Owing to the asymptotic freedom of QCD, the $\mathrm{U}(1)_{A}$ axial symmetry becomes an exact symmetry only for $T \rightarrow \infty$. However, the nature of the chiral phase transition crucially depends on how axial anomaly manifests itself in the two-point correlation functions of light scalar and pseudoscalar mesons for $T \geq T_{c}$. If the isotriplet scalar $\delta$ and the isotriplet pseudoscalar $\pi$ remain nondegenerate at

Published by the American Physical Society under the terms of the Creative Commons Attribution 4.0 International license. Further distribution of this work must maintain attribution to the author(s) and the published article's title, journal citation, and DOI. Funded by SCOAP ${ }^{3}$.
$T \geq T_{c}$, then the chiral phase transition is expected to be of second order, belonging to a three-dimensional $O(4)$ universality class [8]. But if the $\delta$ and $\pi$ become degenerate at $T \geq T_{c}$, then the chiral phase transition can be either first [8] or second order [9-11]. For the physical value of $m_{l}$, the $\delta$ and $\pi$ remain nondegenerate around the chiral crossover [3,12-15]. However, what happens for $T \simeq T_{c}$ as $m_{l} \rightarrow 0$ remains an open question [16-23] due to the lack of stateof-the-art lattice QCD calculations with controlled continuum and chiral extrapolations.

It has been shown that if Dirac eigenvalue spectrum $\rho\left(\lambda, m_{l}\right)$ is an analytic function of $m_{l}^{2}$ and $\lambda$ then in the chiral limit $\mathrm{U}(1)_{A}$ anomaly will not be manifested in differences of up to six-point correlation functions of $\pi$ and $\delta$ that can be connected via a $\mathrm{U}(1)_{A}$ rotation [24]. However, weakly interacting instanton $[25,26]$ gas motivated $\rho \sim m_{l}^{2} \delta(\lambda)$ can lead to nondegeneracy of the twopoint $\pi$ and $\delta$ correlation functions even as $m_{l} \rightarrow 0$ [13]. While the $m_{l}^{2}$ factor naturally arises from the two light fermion determinants, the $\delta(\lambda)$-like structure is motivated by the limit when the small shift from zero to the near-zero modes, resulting from the weak interactions among localized (quasi)instantons and anti-instantons, can be neglected [27,28]. Lattice QCD studies show that, for the physical values of $m_{l}$ and for sufficiently high temperatures, the $T$ dependence of a $\mathrm{U}(1)_{A}$-breaking measure, the topological susceptibility, follows dilute instanton gas approximation 
prediction (for a recent review, see [29]). However, whether these findings arise due to an underlying structure of $\rho \sim m_{l}^{2} \delta(\lambda)$ and what happens for $m_{l} \rightarrow 0$ have remained unanswered. Some lattice QCD studies have observed infrared enhancement in $\rho[13,14,19,30,31]$, however, whether such enhancements scale as $m_{l}^{2}$ as $m_{l} \rightarrow 0$ have not been demonstrated. In other lattice QCD calculations, no infrared enhancement in $\rho$ was observed [17,18,20,22], showing the importance of controlling lattice artifacts through continuum extrapolations. On the other hand, in Ref. [32] it was argued that if $\pi$ and $\delta$ were to remain nondegenerate at $T \geq T_{c}$, then chiral symmetry restoration demands non-Poisson correlations among the infrared eigenvalues.

In this Letter we connect all the above issues: first, by establishing novel relations between $\partial^{n} \rho / \partial m_{l}^{n}$ and correlation among the eigenvalues, then by obtaining $\partial^{n} \rho / \partial m_{l}^{n}$ from state-of-the-art lattice QCD calculations. Finally, we demonstrate how the signature of axial anomaly in two-point $\delta$ and $\pi$ correlation functions arises as $m_{l} \rightarrow 0$.

$\partial^{n} \rho / \partial m_{l}^{n}$ and $\mathrm{U}(1)_{A}$ anomaly.-For $(2+1)$-flavor $\mathrm{QCD}$, the Dirac eigenvalue spectrum is given by

$$
\begin{aligned}
\rho\left(\lambda, m_{l}\right)= & \frac{T}{V Z[\mathcal{U}]} \int \mathcal{D}[\mathcal{U}] e^{-S_{G}[\mathcal{U}]} \operatorname{det}\left[\not D[\mathcal{U}]+m_{s}\right] \\
& \times\left(\operatorname{det}\left[\not D[\mathcal{U}]+m_{l}\right]\right)^{2} \rho_{U}(\lambda) .
\end{aligned}
$$

Here, $\rho_{U}(\lambda)=\sum_{j} \delta\left(\lambda-\lambda_{j}\right), \quad \lambda_{j}$ are the eigenvalues of the massless Dirac matrix $\not D[\mathcal{U}]$ for a given background $\mathrm{SU}(3)$ gauge field $\mathcal{U}, V$ is the spatial volume, $S_{G}[\mathcal{U}]$ is the gauge action, and the partition function $Z[\mathcal{U}]=\int \mathcal{D}[\mathcal{U}] e^{-S_{G}[\mathcal{U}]} \operatorname{det}\left[\not D[\mathcal{U}]+m_{s}\right]\left(\operatorname{det}\left[\not D[\mathcal{U}]+m_{l}\right]\right)^{2}$.

Note that $\rho_{U}(\lambda)$ does not explicitly depend on $m_{l}$, however, $m_{l}$ dependence enters $\rho$ through the integration over the gauge fields. Furthermore,

$$
\begin{aligned}
\operatorname{det}\left[\not \supset[\mathcal{U}]+m_{l}\right] & =\prod_{j}\left(+i \lambda_{j}+m_{l}\right)\left(-i \lambda_{j}+m_{l}\right) \\
& =\exp \left(\int_{0}^{\infty} d \lambda \rho_{U}(\lambda) \ln \left[\lambda^{2}+m_{l}^{2}\right]\right) .
\end{aligned}
$$

Substituting Eq. (2) in Eq. (1) and $Z[\mathcal{U}]$ it is straightforward to obtain $\partial^{n} \rho / \partial m_{l}^{n}$, e.g.,

$$
\begin{aligned}
& \frac{V}{T} \frac{\partial \rho}{\partial m_{l}}=\int_{0}^{\infty} d \lambda_{2} \frac{4 m_{l} C_{2}\left(\lambda, \lambda_{2} ; m_{l}\right)}{\lambda_{2}^{2}+m_{l}^{2}} \\
\frac{V}{T} \frac{\partial^{2} \rho}{\partial m_{l}^{2}}= & \int_{0}^{\infty} d \lambda_{2} \frac{4\left(\lambda_{2}^{2}-m_{l}^{2}\right) C_{2}\left(\lambda, \lambda_{2} ; m_{l}\right)}{\left(\lambda_{2}^{2}+m_{l}^{2}\right)^{2}} \\
& +\int_{0}^{\infty} d \lambda_{2} d \lambda_{3} \frac{\left(4 m_{l}\right)^{2} C_{3}\left(\lambda, \lambda_{2}, \lambda_{3} ; m_{l}\right)}{\left(\lambda_{2}^{2}+m_{l}^{2}\right)\left(\lambda_{3}^{2}+m_{l}^{2}\right)}, \quad \text { with }
\end{aligned}
$$

$$
C_{n}\left(\lambda_{1}, \ldots, \lambda_{n} ; m_{l}\right)=\left\langle\prod_{i=1}^{n}\left[\rho_{U}\left(\lambda_{i}\right)-\left\langle\rho_{U}\left(\lambda_{i}\right)\right\rangle\right]\right\rangle .
$$

The difference of the integrated two-point functions, i.e., susceptibilities, of the isotriplet pseudoscalar, $\pi^{i}(x)=\mathrm{i} \bar{\psi}_{l}(x) \gamma_{5} \tau^{i} \psi_{l}(x)$, and the isotriplet scalar, $\delta^{i}(x)=\bar{\psi}_{l}(x) \tau^{i} \psi_{l}(x)$, mesons is defined as

$$
\chi_{\pi}-\chi_{\delta}=\int d^{4} x\left\langle\pi^{i}(x) \pi^{i}(0)-\delta^{i}(x) \delta^{i}(0)\right\rangle .
$$

For $T \geq T_{c}$ owing to the degeneracy of $\pi$ and the isosinglet scalar meson in the chiral limit [13]

$$
\chi_{\pi}-\chi_{\delta}=\chi_{\mathrm{disc}}
$$

where $\chi_{\text {disc }}$ is the quark-line disconnected part of the isosinglet scalar meson susceptibility [33],

$$
\chi_{\text {disc }}=\frac{T}{V} \int d^{4} x\left\langle[\bar{\psi}(x) \psi(x)-\langle\bar{\psi}(x) \psi(x)\rangle]^{2}\right\rangle .
$$

These $\mathrm{U}(1)_{A}$ symmetry-breaking measures are related to $\rho$ through $[13,32,34]$

$$
\begin{gathered}
\chi_{\pi}-\chi_{\delta}=\int_{0}^{\infty} d \lambda \frac{8 m_{l}^{2} \rho}{\left(\lambda^{2}+m_{l}^{2}\right)^{2}}, \\
\chi_{\text {disc }}=\int_{0}^{\infty} d \lambda \frac{4 m_{l} \partial \rho / \partial m_{l}}{\lambda^{2}+m_{l}^{2}} .
\end{gathered}
$$

In the Poisson limit, $C_{n}$ is given by $C_{n}^{\mathrm{Po}}\left(\lambda_{1}, \ldots, \lambda_{n}\right)=\delta\left(\lambda_{1}-\right.$ $\left.\lambda_{2}\right) \cdots \delta\left(\lambda_{n}-\lambda_{n-1}\right)\left\langle\left(\rho_{U}\left(\lambda_{1}\right)-\left\langle\rho_{U}\left(\lambda_{1}\right)\right\rangle\right)^{n}\right\rangle=\delta\left(\lambda_{1}-\lambda_{2}\right) \cdots$ $\delta\left(\lambda_{n}-\lambda_{n-1}\right)\left\langle\rho_{U}\left(\lambda_{1}\right)\right\rangle+\mathcal{O}(1 / N)$, where $2 N \propto V / T$ is the total number of eigenvalues. In this limit,

$$
\begin{aligned}
& \left(\frac{\partial \rho}{\partial m_{l}}\right)^{\text {Po }}=\frac{4 m_{l} \rho}{\lambda^{2}+m_{l}^{2}}-\frac{V \rho}{T N}\langle\bar{\psi} \psi\rangle, \\
& \left(\frac{\partial^{2} \rho}{\partial m_{l}^{2}}\right)^{\text {Po }}=\frac{4 \rho}{\lambda^{2}+m_{l}^{2}}+\frac{8 m_{l}^{2} \rho}{\left(\lambda^{2}+m_{l}^{2}\right)^{2}}+\frac{2 V^{2} \rho}{T^{2} N^{2}}\langle\bar{\psi} \psi\rangle^{2} \\
& -\frac{V \rho}{T N}\left(\frac{8 m_{l}\langle\bar{\psi} \psi\rangle}{\lambda^{2}+m_{l}^{2}}+2 \chi_{\pi}-\chi_{\delta}\right),
\end{aligned}
$$

where $\langle\bar{\psi} \psi\rangle=(T / V)\left(d \ln Z[\mathcal{U}] / d m_{l}\right)$. In the chiral limit, this leads to $\chi_{\text {disc }}^{\mathrm{Po}}=2\left(\chi_{\pi}-\chi_{\delta}\right)$, in clear violation of the chiral symmetry restoration condition in Eq. (7), unless both sides of the equation trivially vanish.

Lattice QCD calculations. - Lattice QCD calculations were carried out at $T \approx 205 \mathrm{MeV} \approx 1.6 T_{c}$ for $(2+1)$ flavor QCD using the highly improved staggered quarks and the tree-level Symanzik gauge action, a setup extensively used by the HotQCD Collaboration [2,5,35-37]. The $m_{s}$ was tuned to its physical value and three lattice spacings 

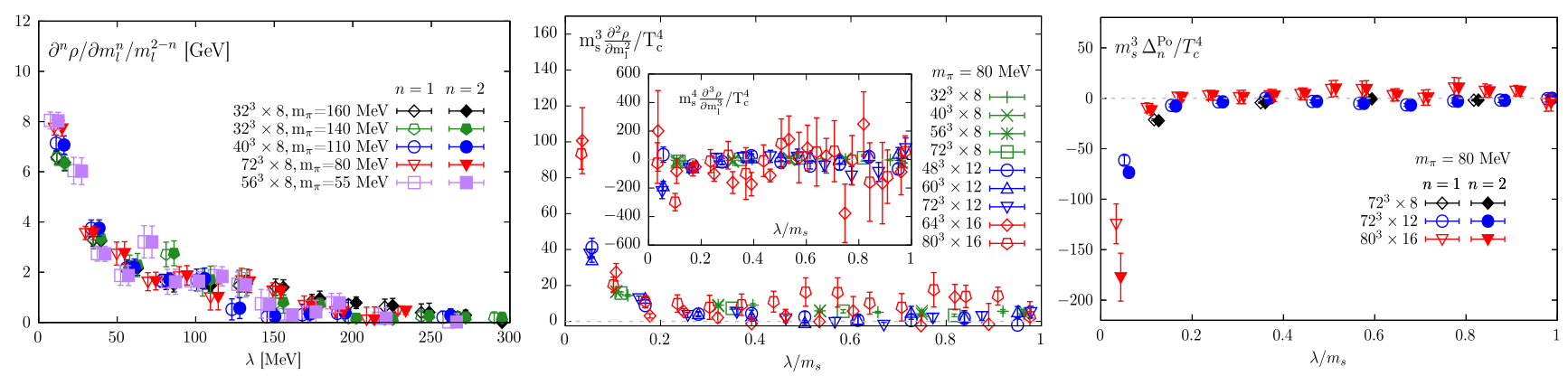

FIG. 1. Left: Light sea quark mass dependence of $m_{l}^{-1} \partial \rho\left(\lambda, m_{l}\right) / \partial m_{l}$ (open symbols) and $\partial^{2} \rho\left(\lambda, m_{l}\right) / \partial m_{l}^{2}$ (filled symbols) using $N_{\tau}=8$ lattices. Middle: Lattice spacing and volume dependence of $\partial^{2} \rho\left(\lambda, m_{l}\right) / \partial m_{l}^{2}$ and $\partial^{3} \rho\left(\lambda, m_{l}\right) / \partial m_{l}^{3}$ (inset) for $m_{\pi}=80 \mathrm{MeV}$. Right: The differences, $\Delta_{n}^{\mathrm{Po}}=m_{l}^{n-2}\left[\partial^{n} \rho / \partial m_{l}^{n}-\left(\partial^{n} \rho / \partial m_{l}^{n}\right)^{\mathrm{Po}}\right]$ [cf. Eqs. (11) and (12)], for $m_{\pi}=80 \mathrm{MeV}$ and three lattice spacings. In all cases, results are obtained at $T \approx 205 \mathrm{MeV}$ and the filled symbols have been slightly shifted horizontally for visibility.

$a=\left(T N_{\tau}\right)^{-1}=0.12,0.08,0.06 \mathrm{fm}$, corresponding to lattice temporal extents $N_{\tau}=8,12,16$, were used [15]. Calculations were done with $m_{l}=m_{s} / 20, m_{s} / 27$, $m_{s} / 40, m_{s} / 80, m_{s} / 160$ that correspond to $m_{\pi} \simeq 160$, $140,110,80,55 \mathrm{MeV}$, respectively. The spatial extents $\left(N_{\sigma}\right)$ of the lattices were chosen to have aspect ratios in the range of $N_{\sigma} / N_{\tau}=4-9$. The gauge field configurations were generated using the rational hybrid Monte Carlo algorithm [38,39]. Gauge configurations from every 10th molecular dynamics trajectory of unit length were saved to carry out various measurements. $\rho$ and $C_{n}$ were computed by measuring $\rho_{U}(\lambda)$ over the entire range of $\lambda$ using the Chebyshev filtering technique combined with the stochastic estimate method [40-44] on 2000 configurations. Orders of the Chebyshev polynomials were chosen to be $(1-5) \times 10^{5}$ and 24 Gaussian stochastic sources were used. Measurements of $\chi_{\text {disc }}$ and $\chi_{\pi}-\chi_{\delta}$ were done by inverting the light fermion matrix using 50 Gaussian random sources on 2000-10 000 configurations [45].

Results.-Figure 1 (left) shows the $m_{l}$ dependence of $m_{l}^{-1} \partial \rho / \partial m_{l}$ and $\partial^{2} \rho / \partial m_{l}^{2}$ at $T \approx 1.6 T_{c}$, obtained for lattices with $N_{\tau}=8$ and the largest available $N_{\sigma}$ for that $m_{l}$. We observe that $m_{l}^{-1}\left(\partial \rho / \partial m_{l}\right)$ and $\partial^{2} \rho / \partial m_{l}^{2}$ are almost identical and independent of $m_{l}$. Also, $m_{l}^{-1} \partial \rho / \partial m_{l}$ and $\partial^{2} \rho / \partial m_{l}^{2}$ are peaked at $\lambda \rightarrow 0$ and drop rapidly toward zero for $\lambda / T \gtrsim 1$. Figure 1 (middle) depicts the lattice spacing and volume dependence of $\partial^{2} \rho / \partial m_{l}^{2}$ and $\partial^{3} \rho / \partial m_{l}^{3}$ for $m_{\pi}=80 \mathrm{MeV}$. To compare these quantities across different lattice spacings, we multiply with the appropriate powers of $m_{s}$ to make them renormalization group invariant and make them dimensionless by rescaling with appropriate powers of $T_{c}=132 \mathrm{MeV}$. We see that the peaked structure in $\partial^{2} \rho / \partial m_{l}^{2}$ at $\lambda \rightarrow 0$ becomes sharper as $a \rightarrow 0$ and shows little volume dependence (see Supplemental Material [46]). Moreover, within errors, $\partial^{3} \rho / \partial m_{l}^{3}$ are found to be consistent with zero in all the cases. The findings $m_{l}^{-1} \partial \rho / \partial m_{l} \approx \partial^{2} \rho / \partial m_{l}^{2}$ and $\partial^{3} \rho / \partial m_{l}^{3} \approx 0$ show that the peaked structure $\rho\left(\lambda \rightarrow 0, m_{l} \rightarrow 0\right) \propto m_{l}^{2}$. In Fig. 1 (right) we show the difference $\Delta_{n}^{\text {Po }}=$ $m_{l}^{n-2}\left[\partial^{n} \rho / \partial m_{l}^{n}-\left(\partial^{n} \rho / \partial m_{l}^{n}\right)^{\mathrm{Po}}\right] \quad(n=1, \quad 2)$, with the
Poisson approximations for $\partial^{n} \rho / \partial m_{l}^{n}$ as defined in Eqs. (11) and (12). The fact $\Delta_{n}^{\mathrm{Po}}<0$ shows that the repulsive non-Poisson correlation within the small $\lambda$ gives rise to the $\rho(\lambda \rightarrow 0)$ peak.

In Fig. 2 we show that $\rho$ and $\partial \rho / \partial m_{l}$ reproduce directly measured $\chi_{\pi}-\chi_{\delta}$ and $\chi_{\text {disc }}$ using Eqs. (9) and (10), respectively. The numerical integrations in $\lambda$ were performed using the rectangle method, where the largest value of $\lambda$ was estimated using the power method and the statistical error of integration was obtained using the jackknife method. Since we saw very mild volume dependence in all the quantities, we only present results from the largest available volume for each $N_{\tau}$ and $m_{l}$. We checked
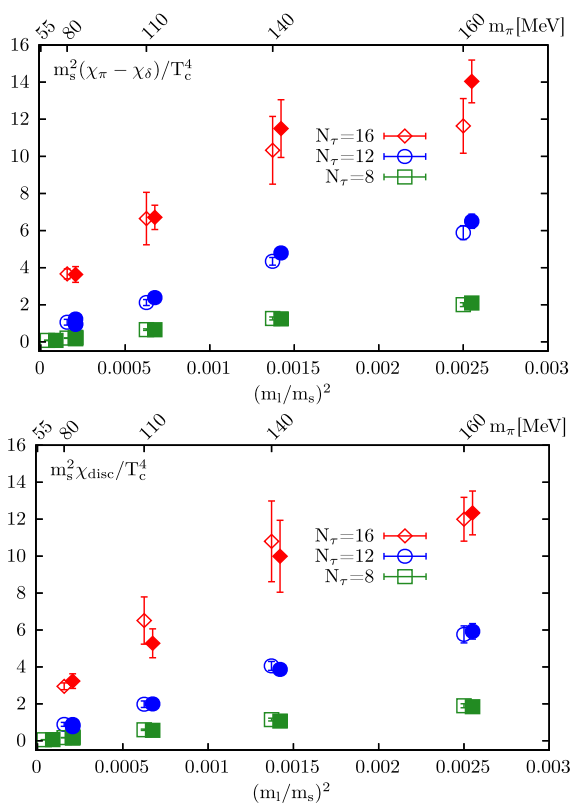

FIG. 2. Comparisons of direct measurements (open symbols) of $\chi_{\pi}-\chi_{\delta}$ (top) and $\chi_{\text {disc }}$ (bottom) with those reconstructed (filled symbols, slightly shifted horizontally for visibility) from $\rho$ [cf. Eq. (9)] and $\partial \rho / \partial m_{l}$ [cf. Eq. (10)], respectively. The results are shown for all values of light quark masses and lattice spacings at $T \approx 205 \mathrm{MeV}$. 

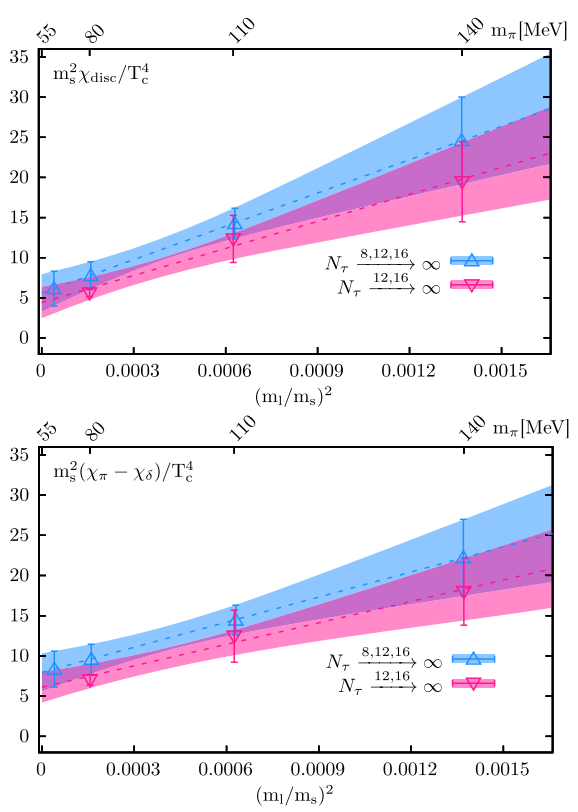

FIG. 3. Continuum and chiral extrapolated results for $\chi_{\text {disc }}$ (top) and $\chi_{\pi}-\chi_{\delta}$ (bottom) at $T \approx 205 \mathrm{MeV}$. See text for details.

that only the infrared $\lambda / T \lesssim 1$ parts of $\rho$ and $\partial \rho / \partial m_{l}$ are needed for the reproductions of $\chi_{\pi}-\chi_{\delta}$ and $\chi_{\text {disc }}$, within errors, for all $N_{\tau}$ and $m_{l}$. Additionally, we checked that once the bin size of $\lambda$ in the numerical integration of Eq. (9) is chosen to reproduce directly measured $\chi_{\pi}-\chi_{\delta}$, the same bin size automatically reproduces $\chi_{\text {disc }}$ and $\langle\bar{\psi} \psi\rangle$ without any further tuning. We observe that both $\chi_{\text {disc }}$ and $\chi_{\pi}-\chi_{\delta}$ are linear in $m_{l}^{2}$ for all lattice spacings and especially for $m_{\pi} \lesssim 140 \mathrm{MeV}$; this is in accord with the expectation $Z[\mathcal{U}]$ is an even function of $m_{l}$ for $T \geq T_{c}$ due to the restoration of the $Z(2)$ subgroup of $\mathrm{SU}(2)_{L} \times \mathrm{SU}(2)_{R}$.

In Fig. 3 we show the continuum and chiral extrapolated results for $\chi_{\text {disc }}$ and $\chi_{\pi}-\chi_{\delta}$. Using all the data for $N_{\tau}=8$, 12, 16 and $m_{\pi} \leq 140 \mathrm{MeV}$, we performed a joint $a, m_{l} \rightarrow 0$ extrapolation of the form $\chi_{\text {disc }}\left(a, m_{l}\right)=$ $\chi_{\text {disc }}(0,0)+a_{1} / N_{\tau}^{2}+a_{2} / N_{\tau}^{4}+\left(m_{l} / m_{s}\right)^{2}\left[b_{0}+b_{1} / N_{\tau}^{2}+\right.$ $\left.b_{2} / N_{\tau}^{4}\right]$. Fits were performed on each bootstrap sample of the data set. The bootstrap samples were created by randomly choosing data from Gaussian distributions with means equal to the average values and variances equal to the $1-\sigma$ errors of the directly measured $\chi_{\text {disc }}$. We chose the median value of the resulting bootstrap distribution as the final result (depicted by the upward triangles) and the $68 \%$ percentiles confidence interval of the resulting distribution as the errors on the final results (the band labeled by $\left.N_{\tau} \stackrel{8,12,16}{\longrightarrow} \infty\right)$. Since we used the so-called rooted-staggered formulation [53-56] for our (2+1)-flavor lattice QCD, we also checked that the same $\chi_{\text {disc }}(0,0)$ is obtained within errors by first carrying out the $a \rightarrow 0$ extrapolations for each $m_{l}$ and then performing the $m_{l} \rightarrow 0$ extrapolation using the $a \rightarrow 0$ extrapolated results. For this purpose, we used the $N_{\tau}=12,16$ data for each of $m_{l}=$ $m_{s} / 27, m_{s} / 40, m_{s} / 80$ to obtain $\chi_{\text {disc }}\left(0, m_{l}\right)$ by fitting to the ansatz $\chi_{\text {disc }}\left(a, m_{l}\right)=\chi_{\text {disc }}\left(0, m_{l}\right)+d_{1} / N_{\tau}^{2}$. Then the chiral extrapolation was carried out using $\chi_{\text {disc }}\left(0, m_{l}\right)=$ $\chi_{\text {disc }}(0,0)+d_{2}\left(m_{l} / m_{s}\right)^{2}$ based on the continuum estimates of $\chi_{\text {disc }}\left(0, m_{l}\right)$. These extrapolations were done by using the same bootstrap procedure described before and the final results are indicated with the label $N_{\tau} \stackrel{12,16}{\longrightarrow} \infty$. Exactly the same procedures were followed also for $\chi_{\pi}-\chi_{\delta}$ to obtain its continuum and chiral extrapolated values. After carrying out continuum and chiral extrapolations, we find that Eq. (7) is satisfied within errors, and $\chi_{\text {disc }}$ and $\chi_{\pi}-\chi_{\delta}$ are nonvanishing at a confidence level above $95 \%$.

Conclusions. - In this Letter we establish relations between $\partial^{n} \rho / \partial m_{l}^{n}$ and $C_{n+1}$. To the best of our knowledge, these relations are new in the literature. Based on these relations, for the first time, we present direct computations of $\partial^{n} \rho / \partial m_{l}^{n}$ employing state-of-the-art lattice QCD techniques. The results presented in this Letter led us to conclude that, in chiral symmetric $(2+1)$-flavor QCD at $T \approx 1.6 T_{c}$, (i) $\rho\left(\lambda \rightarrow 0, m_{l}\right)$ develops a peaked structure due to repulsive non-Poisson correlations within small $\lambda$; the peak becomes sharper as $a \rightarrow 0$, and its amplitude is $\propto m_{l}^{2}$. (ii) The underlying presence of this $\rho\left(\lambda \rightarrow 0, m_{l}\right)$ leads to manifestations of $\mathrm{U}(1)_{A}$ anomaly in $\chi_{\pi}-\chi_{\delta}$ and $\chi_{\text {disc }}$. (iii) Axial anomaly remains manifested in $\chi_{\pi}-\chi_{\delta}$ and $\chi_{\text {disc }}$ even in the chiral limit. These suggest that for $T \gtrsim 1.6 T_{c}$ the microscopic origin of axial anomaly is driven by the weakly interacting (quasi)instanton gas motivated $\rho\left(\lambda \rightarrow 0, m_{l} \rightarrow 0\right) \sim m_{l}^{2} \delta(\lambda)$, and the chiral phase transition in $(2+1)$-flavor QCD is of the threedimensional $O(4)$ universality class.

The above conclusions are based on the continuum extrapolated lattice QCD calculations using the $(2+1)$ flavors of staggered fermions. Confirmations of these continuum extrapolated results using other fermion actions, especially using chiral fermions, are needed in future. Even in those future calculations it will be very difficult to directly identify a structure like $m_{l}^{2} \delta(\lambda)$ in $\rho$ itself as $m_{l} \rightarrow 0$. The formalism developed and techniques presented in this Letter for directly accessing $\partial^{n} \rho / \partial m_{l}^{n}$ will be essential for those future studies too. The same or similar formalism also may have many potential applications beyond the present physics problem: few plausible examples testing the predictions of random matrix theory $[34,57,58]$, determination of strong coupling constant using Dirac eigenvalue spectrum [59], determinations of mass anomalous dimensions in different theories [47,60-63], etc.

This material is based upon work supported by the National Natural Science Foundation of China under Grants No. 11775096, No. 11535012, and No. 11947237; the U.S. Department of Energy, Office of Science, Office of Nuclear Physics through the Award No. DE-SC0012704; the U.S. Department of Energy, Office of Science, Office of 
Nuclear Physics and Office of Advanced Scientific Computing Research within the framework of Scientific Discovery through Advance Computing (SciDAC) award Computing the Properties of Matter with Leadership Computing Resources; and RIKEN Special Postdoctoral Researcher program and JSPS KAKENHI Grant No. JP20K14479. Computations for this work were carried out on the GPU clusters of the Nuclear Science Computing Center at Central China Normal University $\left(\mathrm{NSC}^{3}\right)$, Wuhan, China, and facilities of the USQCD Collaboration, which are funded by the Office of Science of the U.S. Department of Energy. For generating the gauge configurations, the HotQCD software suite was used, and the eigenvalue measurement code was developed also based on the same software suite. We are indebted to the HotQCD Collaboration for sharing their software suite with us.

*yuzhang@mails.ccnu.edu.cn

[1] Y. Aoki, S. Borsanyi, S. Durr, Z. Fodor, S. D. Katz, S. Krieg, and K. K. Szabo, J. High Energy Phys. 06 (2009) 088.

[2] A. Bazavov et al., Phys. Rev. D 85, 054503 (2012).

[3] T. Bhattacharya, M. I. Buchoff, N. H. Christ, H.-T. Ding, R. Gupta et al., Phys. Rev. Lett. 113, 082001 (2014).

[4] C. Bonati, M. D’Elia, M. Mariti, M. Mesiti, F. Negro, and F. Sanfilippo, Phys. Rev. D 92, 054503 (2015).

[5] A. Bazavov et al. (HotQCD Collaboration), Phys. Lett. B 795, 15 (2019).

[6] S. Borsanyi, Z. Fodor, J. N. Guenther, R. Kara, S. D. Katz, P. Parotto, A. Pasztor, C. Ratti, and K. K. Szabo, Phys. Rev. Lett. 125, 052001 (2020).

[7] H. T. Ding et al., Phys. Rev. Lett. 123, 062002 (2019).

[8] R. D. Pisarski and F. Wilczek, Phys. Rev. D 29, 338 (1984).

[9] A. Butti, A. Pelissetto, and E. Vicari, J. High Energy Phys. 08 (2003) 029.

[10] A. Pelissetto and E. Vicari, Phys. Rev. D 88, 105018 (2013).

[11] M. Grahl, Phys. Rev. D 90, 117904 (2014).

[12] M. Cheng et al., Eur. Phys. J. C 71, 1564 (2011).

[13] A. Bazavov, T. Bhattacharya, M. I. Buchoff, M. Cheng, N. H. Christ et al. (HotQCD Collaboration), Phys. Rev. D 86, 094503 (2012).

[14] M. I. Buchoff, M. Cheng, N. H. Christ, H. T. Ding, C. Jung et al., Phys. Rev. D 89, 054514 (2014).

[15] A. Bazavov, S. Dentinger, H. T. Ding, P. Hegde, O. Kaczmarek et al., Phys. Rev. D 100, 094510 (2019).

[16] H. Ohno, U. Heller, F. Karsch, and S. Mukherjee, Proc. Sci., LATTICE2012 (2012) 095 [arXiv:1211.2591].

[17] G. Cossu, S. Aoki, H. Fukaya, S. Hashimoto, T. Kaneko, H. Matsufuru, and J. I. Noaki, Phys. Rev. D 87, 114514 (2013).

[18] T.-W. Chiu, W.-P. Chen, Y.-C. Chen, H.-Y. Chou, and T.-H. Hsieh (TWQCD Collaboration), Proc. Sci., LATTICE2013 (2014) 165 [arXiv:1311.6220].

[19] V. Dick, F. Karsch, E. Laermann, S. Mukherjee, and S. Sharma, Phys. Rev. D 91, 094504 (2015).

[20] A. Tomiya, G. Cossu, S. Aoki, H. Fukaya, S. Hashimoto, T. Kaneko, and J. Noaki, Phys. Rev. D 96, 034509 (2017); 96, 079902(E) (2017).
[21] B. B. Brandt, A. Francis, H. B. Meyer, O. Philipsen, D. Robaina, and H. Wittig, J. High Energy Phys. 12 (2016) 158.

[22] K. Suzuki, S. Aoki, Y. Aoki, G. Cossu, H. Fukaya, S. Hashimoto, and C. Rohrhofer (JLQCD Collaboration), Proc. Sci., LATTICE2019 (2020) 178 [arXiv:2001.07962].

[23] S. Aoki, Y. Aoki, G. Cossu, H. Fukaya, S. Hashimoto, T. Kaneko, C. Rohrhofer, and K. Suzuki (JLQCD Collaboration), arXiv:2011.01499.

[24] S. Aoki, H. Fukaya, and Y. Taniguchi, Phys. Rev. D 86, 114512 (2012).

[25] G. 't Hooft, Phys. Rev. Lett. 37, 8 (1976).

[26] G.'t Hooft, Phys. Rev. D 14, 3432 (1976); 18, 2199(E) (1978).

[27] D. J. Gross, R. D. Pisarski, and L. G. Yaffe, Rev. Mod. Phys. 53, 43 (1981).

[28] T. Kanazawa and N. Yamamoto, Phys. Rev. D 91, 105015 (2015).

[29] M. P. Lombardo and A. Trunin, Int. J. Mod. Phys. A 35, 2030010 (2020).

[30] A. Alexandru and I. Horváth, Phys. Rev. D 92, 045038 (2015).

[31] A. Alexandru and I. Horváth, Phys. Rev. D 100, 094507 (2019).

[32] T. Kanazawa and N. Yamamoto, J. High Energy Phys. 01 (2016) 141.

[33] The term $\langle\bar{\psi} \psi\rangle^{2}$ arises naturally from $\partial\langle\bar{\psi} \psi\rangle / \partial m_{l}$ and trivially vanishes for $T \geq T_{c}$ as $m_{l} \rightarrow 0$. Following standard convention, this term was subtracted off to cancel the $\left(m_{l} / a\right)^{2}$ divergence.

[34] D. Toublan and J. J. M. Verbaarschot, Nucl. Phys. B603, 343 (2001).

[35] A. Bazavov, T. Bhattacharya, C. DeTar, H. T. Ding, S. Gottlieb et al. (HotQCD Collaboration), Phys. Rev. D 90, 094503 (2014).

[36] A. Bazavov, T. Bhattacharya, C. E. DeTar, H. T. Ding, S. Gottlieb et al. (HotQCD Collaboration), Phys. Rev. D 86, 034509 (2012).

[37] A. Bazavov, H. T. Ding, P. Hegde, O. Kaczmarek, F. Karsch et al., Phys. Rev. D 95, 054504 (2017).

[38] M. A. Clark, A. D. Kennedy, and Z. Sroczynski, Nucl. Phys. B, Proc. Suppl. 140, 835 (2005).

[39] A. Bazavov et al. (MILC Collaboration), Phys. Rev. D 82, 074501 (2010).

[40] H.-T. Ding, O. Kaczmarek, F. Karsch, S.-T. Li, S. Mukherjee, A. Tomiya, and Y. Zhang, Proc. Sci., LATTICE2019 (2020) 251 [arXiv:2001.05217].

[41] L. Giusti and M. Luscher, J. High Energy Phys. 03 (2009) 013.

[42] G. Cossu, H. Fukaya, S. Hashimoto, T. Kaneko, and J.-I. Noaki, Prog. Theor. Exp. Phys. 2016, 093 B06 (2016).

[43] Z. Fodor, K. Holland, J. Kuti, S. Mondal, D. Nogradi, and C. H. Wong, Proc. Sci., LATTICE2015 (2016) 310 [arXiv:1605.08091].

[44] H.-T. Ding, S.-T. Li, A. Tomiya, X.-D. Wang, and Y. Zhang, arXiv:2008.00493.

[45] $\chi_{\pi}$ was obtained through the Ward identity $m_{l} \chi_{\pi}=\langle\bar{\psi} \psi\rangle$ $[44,56]$ and $\chi_{\delta}$ is the connected chiral susceptibility [14]. We checked that these results were reproduced, within errors, by the susceptibilities of the independently computed $\pi$ and $\delta$ two-point correlation functions. 
[46] See Supplemental Material at http://link.aps.org/ supplemental/10.1103/PhysRevLett.126.082001 for the technical details of this study, which includes Refs. [2,5,32,35-37,40-44,47,48,50-52]. We also found that even when the same bin size in $\lambda / m_{s}$ is used for all three lattice spacings the infrared peak structure persists as continuum limit is approached, see Fig. S8 of the Supplemental Material.

[47] A. Patella, Phys. Rev. D 86, 025006 (2012).

[48] A. Ramos and S. Sint, Eur. Phys. J. C 76, 15 (2016).

[49] E. Itou and A. Tomiya, Proc. Sci., LATTICE2014 (2014) 252 [arXiv:1411.1155].

[50] P. de Forcrand and B. Jager, EPJ Web Conf. 175, 14022 (2018).

[51] E. D. Napoli, E. Polizzi, and Y. Saad, arXiv:1308.4275.

[52] Y. Saad, Numerical Methods for Large Eigenvalue Problems (Society for Industrial and Applied Mathematics, Philadelphia, 2011).
[53] C. Bernard, Phys. Rev. D 71, 094020 (2005).

[54] C. Bernard, M. Golterman, Y. Shamir, and S. R. Sharpe, Phys. Lett. B 649, 235 (2007).

[55] S. R. Sharpe, Proc. Sci., LAT2006 (2006) 022 [arXiv:heplat/0610094].

[56] G. W. Kilcup and S. R. Sharpe, Nucl. Phys. B283, 493 (1987).

[57] J. Osborn and J. Verbaarschot, Nucl. Phys. B525, 738 (1998).

[58] A. S. Christensen, K. Splittorff, and J. Verbaarschot, J. High Energy Phys. 11 (2014) 113.

[59] K. Nakayama, H. Fukaya, and S. Hashimoto, Phys. Rev. D 98, 014501 (2018).

[60] A. Patella, Phys. Rev. D 84, 125033 (2011).

[61] K. Cichy, J. High Energy Phys. 08 (2014) 127.

[62] A. Cheng, A. Hasenfratz, G. Petropoulos, and D. Schaich, J. High Energy Phys. 07 (2013) 061.

[63] N. Karthik and R. Narayanan, Phys. Rev. Lett. 125, 261601 (2020). 